\title{
Mitigating the geometrical limitations of conventional sputtering by controlling the ion- to-neutral ratio during high power pulsed magnetron sputtering
}

Grzegorz Greczynski, Jens Jensen and Lars Hultman

\section{Linköping University Post Print}

N.B.: When citing this work, cite the original article.

Original Publication:

Grzegorz Greczynski, Jens Jensen and Lars Hultman, Mitigating the geometrical limitations of conventional sputtering by controlling the ion-to-neutral ratio during high power pulsed magnetron sputtering, 2011, Thin Solid Films, (519), 19, 6354-6361.

http://dx.doi.org/10.1016/j.tsf.2011.04.031

Copyright: Elsevier

http://www.elsevier.com/

Postprint available at: Linköping University Electronic Press

http://urn.kb.se/resolve?urn=urn:nbn:se:liu:diva-69790 


\title{
Mitigating the geometrical limitations of conventional sputtering by controlling the ion-to-neutral ratio during high power pulsed magnetron sputtering
}

\author{
G. Greczynski, J. Jensen and L. Hultman \\ Department of Physics, Linköping University, 58331 Linköping, Sweden
}

\begin{abstract}
High power pulsed magnetron sputtering has been used to grow thin chromium layers on substrates facing and orthogonal to the target. It is demonstrated that at low peak target current density, $j_{T}<0.6 \mathrm{~A} / \mathrm{cm}^{2}$ corresponding to a low ion-to-neutral flux ratio, films grown on substrates facing the target exhibit in-plane alignment. This is due to the rectangular shape of the target that yields an asymmetry in the off-normal flux of sputtered species. With increasing $j_{T}$ the biaxial alignment degrades, as the major portion of the incoming flux (ions) can be effectively steered by the electric field of the substrate to remove asymmetry imposed by geometrical restrictions. Eventually, at $j_{T}=1.7 \mathrm{~A} / \mathrm{cm}^{2}$ a fiber texture is obtained. For films grown on substrates orthogonal to the target, the large column tilt characteristic for growth at low $j_{T}$, decreases with increasing ion content in the flux and almost disappears at the highest value of $j_{T}$. The latter indicates that material flux to the substrate is highly ionized so that deposition takes place along substrate normal despite the high nominal inclination angle. Thus, in the limit of high $j_{T}$ the artifacts of conventional physical vapor deposition, resulting from the line-of-sight deposition, are effectively eliminated and the film growth proceeds more or less unaffected by the substrate orientation. Samples mounted orthogonally thus possess a similar texture, morphology, and topography as those facing the target.
\end{abstract}

Keywords: HIPIMS; HPPMS; chromium; ionized PVD 


\section{INTRODUCTION}

Thin films prepared by magnetron sputtering often have a fiber texture with pronounced out-of-plane orientation and random in-plane (azimuthal) distribution of crystalline grains. Under some circumstances a biaxial alignment can be obtained, in which case apart from common alignment along the surface normal, crystallites possess an in-plane preferred orientation what makes them similar to single-crystal films [1]. There are two reasons for the biaxial orientation to occur, both relying on lowering the lateral symmetry of incident material flux during growth: ion beam assisted deposition and inclined substrate deposition. In the first case the off-normal ion bombardment restricts the azimuthal distribution, which is believed to result from the fact that grains oriented in a way that facilitates channeling of the ion beam (with open crystallographic planes parallel to the ion beam) have lower sputtering yield and overgrow misaligned grains [2]. In the case of deposition performed on inclined substrates, the off-normal material flux leads to grains with the in-plane orientation resulting in the highest capture crosssection for diffusing ad-atoms [1].

Apart from bi-axial alignment, films deposited on substrates not facing the target often exhibit pronounced column tilt in the direction of incoming flux. The column tilt angle $\gamma$ (relative to the surface normal) is smaller than the angle defined by the direction of incoming flux $(\delta)$. The effect of column tilt that satisfies $\gamma<\delta$ has been observed for $\mathrm{Cr}$ films deposited by direct current magnetron sputtering (DCMS) on obliquely-mounted substrates $[3,4]$ and is believed to result from the conservation of the momentum component parallel to the film surface [5]. Surface 
diffusion also plays a role such that columns tend to tilt back towards the vapor incident direction. Hence, the so-called "tangent rule" applies [6] that relates both angles through

$$
2 \tan \gamma=\tan \delta
$$

This empirical relationship holds best for $0^{\circ} \leq \delta \leq 60^{\circ}[7]$.

Since bi-axial alignment and column tilt are related to the geometry of the targetsubstrate configuration and the line-of-sight nature of conventional physical vapor deposition (PVD) processing they can not be easily avoided. Alami et al. [8], however, demonstrated that the column tilt of Ta films on surfaces orthogonal to the target can be eliminated by using high power pulsed magnetron sputtering (HIPIMS or HPPMS) $[9,10]$. While films prepared by HIPIMS had columns growing along surface normal, DCMS films showed pronounced column tilt towards the direction of incident material flux. The effect was explained by high ionization of the sputtered flux in the case of HIPIMS. In line with these findings Böhlmark et al. [11] showed that the spatial distribution of material flux during HIPIMS can be steered with an external magnetic field, due to high degree of ionized species in the sputtered material.

The effect of HIPIMS on the occurrence of geometry-induced biaxial alignment has not been addressed. Moreover, a systematic study on the relationship between the relative ion content in the sputtered flux and resulting column tilt is lacking. Therefore, in this work we deposit a series of Cr metal films by HIPIMS to investigate (i) the in-plane alignment caused by the off-normal flux of sputtered species and ii) column tilt in films grown on substrates 
orthogonal to the target. We show that the $\mathrm{Cr}$ ion-to-neutral ratio in the material flux incident upon the substrate is a function of the peak target current density and present the influence of the latter parameter on the development of biaxial texture and column tilt.

\section{EXPERIMENTAL DETAILS}

Depositions of Cr films were performed in a CC800/9 coating system manufactured by CemeCon AG in Germany [12]. A single $88 \times 500 \mathrm{~mm}^{2}$ rectangular $\mathrm{Cr}$ target was used. Films were deposited onto $\mathrm{Si}(001)$ substrates cleaned in acetone and isopropanol. The details of the experimental set up are illustrated in Fig. 1. The sample holder was fixed in front of the sputtering target, in the middle of the target height and symmetrically in between both racetracks. Two films were grown in each deposition sequence, one on the substrate facing the target (sample "A" in the X-Z plane) and one on the substrate tilted by $90^{\circ}$ with respect to the surface of the target (sample "B" in the Y-Z plane). No external heating was used and the substrate temperature is estimated to be $\leq 100{ }^{\circ} \mathrm{C}$ due to heating from the plasma. The average power was $3 \mathrm{~kW}$ and the pulsing frequency was varied from $100 \mathrm{~Hz}$ to $1000 \mathrm{~Hz}$ resulting in a peak target current density, $j_{T}$, between $0.1 \mathrm{~A} / \mathrm{cm}^{2}$ and $1.7 \mathrm{~A} / \mathrm{cm}^{2}$. Since the texture of thin films may be a function of film thickness, special care was taken to keep the latter constant. Thus, lowering of the deposition rate with increasing $j_{T}$ was accounted for by increasing the deposition time ${ }^{13}$.

Cross-sectional electron microscopy investigations were carried out on fractured films using LEO 1550 FEG scanning electron microscope. Pole figures were measured with a Philips X'Pert MRD X-ray diffractometer using point-focused $\mathrm{Cu} \mathrm{K} \alpha$ radiation. For each sample, 
three pole figures $\{110\},\{200\}$, and $\{210\}$ were recorded, in addition the measurement of the background signal in the vicinity of the 110 peak was performed. No lateral variation of polar figure plots was detected on any of the samples. The measurements of the ion flux intensity as a function of the peak target current density were performed in the time-resolved mode with a PSM003 mass spectrometer from Hiden Analytical, UK. The ion energy distribution functions during the active phase of the HIPIMS discharge were measured for $\mathrm{Ar}^{+}, \mathrm{Ar}^{2+}, \mathrm{Cr}^{+}$, and $\mathrm{Cr}^{2+}$ ions. The details of the experimental set up, data acquisition, and evaluation procedure is described in detail elsewhere [14].

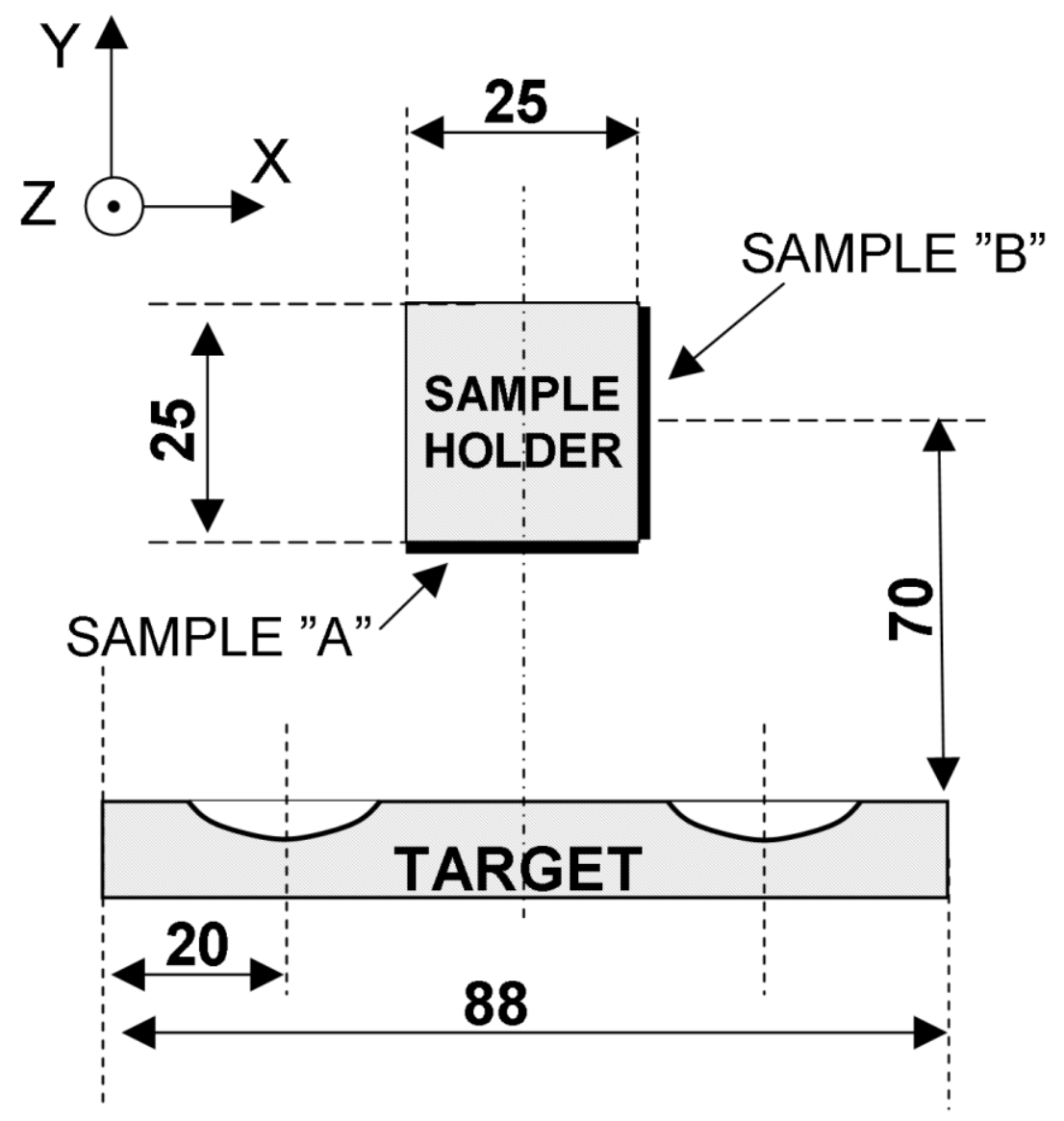

Fig. 1 Projected view of the experimental set-up in cross section along the shorter side of the sputtering target. All dimensions are in millimetres. 
Rutherford Backscattering Spectrometry (RBS) measurements were used to obtain the atomic areal density $\left(\right.$ atoms $/ \mathrm{cm}^{2}$ ) of the films. This was performed using a $2.0 \mathrm{MeV}{ }^{4} \mathrm{He}^{+}$ beam with a backscattering angle of $172^{\circ}$. The incident angle was $7^{\circ}$ with respect to the surface normal (to avoid channeling effects in the substrate). The obtained spectra were evaluated using the SIMNRA 6.05 code [15]. Additional time-of-flight elastic recoil detection analysis (ToFERDA) was made to better evaluate the content of light elements contamination. For this a 40 $\mathrm{MeV}^{127} \mathrm{I}^{9+}$ beam was directed to the films at an incident angle of $67.5^{\circ}$ with respect to the surface normal, and the recoils were detected at an angle of $45^{\circ}$. Both RBS and ToF-ERDA measurements were carried out at the tandem accelerator of Uppsala University, Sweden.

\section{RESULTS AND DISCUSSION}

\section{A) Ion-to-neutral ratio as a function of the peak target current density}

It has been recognized since the early days of HIPIMS that high peak current densities reaching a few $\mathrm{A} / \mathrm{cm}^{2}$ result in significant ionization of sputter-ejected material flux, that largely exceeds that encountered during DCMS. The intensity of ion flux and energy distribution of constituting ions varies in time in tact with the pulsing frequency of target voltage. The energy transfer to the growing film surface is highest during the short ( 100 $\mu$ s) active phase of the discharge (pulse ON) when the vast majority of sputtered particles gets deposited. This is followed by a ms-long period of low intensity plasma when the ion flux to the working piece is well below DCMS standards. In Fig. 2a the ion flux averaged over the $200 \mu$ s long HIPIMS pulse for single- $\left(\mathrm{Cr}^{+}, \mathrm{Ar}^{+}\right)$and doubly- $\left(\mathrm{Cr}^{2+}, \mathrm{Ar}^{2+}\right)$ charged ions is plotted as a function of the peak 

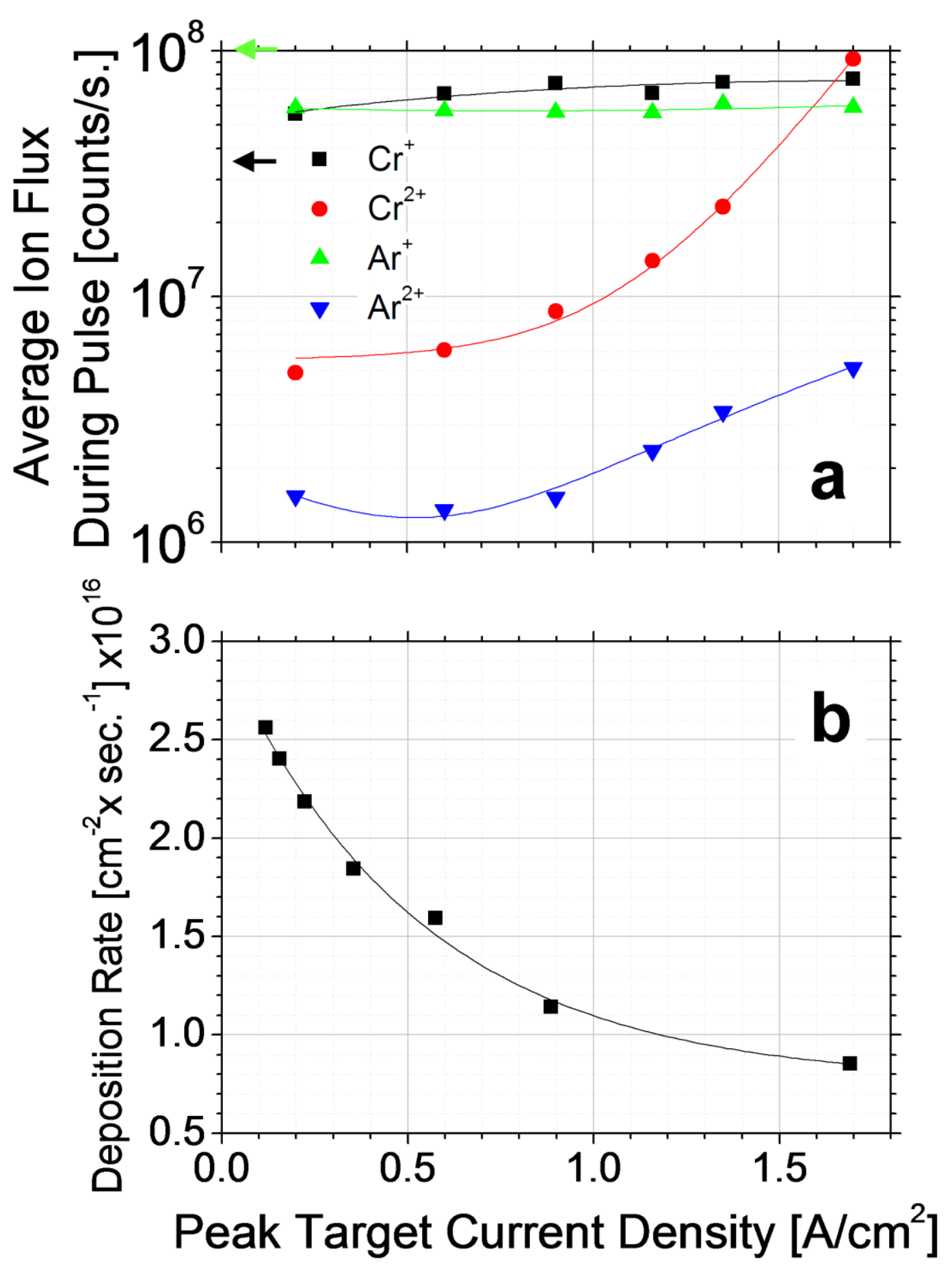

Fig. 2 Average ion flux during the peak phase of the HIPIMS discharge (a) and film growth rate (b) as a function of the peak target current density, $j_{T}$. Arrows in the upper graph indicate DCMS values, note that the DCMS ion count for $\mathrm{Cr}^{2+}$ and $\mathrm{Ar}^{2+}$ ions is out of bounds $\left(2.01 \times 10^{5}\right.$ and $4.02 \times 10^{5} \mathrm{cps}$, respectively). 
target current density, $j_{T}$. The positive ions with energies up to $60 \mathrm{eV}$ are included in this comparison. It is clear from the data that the ion flux during the active phase differs substantially from that of DCMS (corresponding values are indicated with arrows in Fig. 2a). Already at the lowest value of $j_{T}$ the metallic character of HIPIMS plasma is evident. The flux of $\mathrm{Cr}^{+}$ions is increased with respect to DCMS (2-3 times) whereas the average $\mathrm{Ar}^{+}$flux during the active HIPIMS phase is $\sim 50 \%$ lower than that of DCMS. The latter can be attributed to severe gas rarefaction commonly observed during HIPIMS [16]. In consequence for $j_{T}>0.3 \mathrm{~A} / \mathrm{cm}^{2}$, metal ions dominate the flux to the substrate. There is also a high flux of doubly charged $\mathrm{Cr}^{2+}$ ions, that are almost absent in the case of DCMS (only $2 \times 10^{5} \mathrm{cps}$ ). Upon increasing the peak target current density the metal ion flux to the substrate increases. The most prominent change upon going from $j_{T}=0.2 \mathrm{~A} / \mathrm{cm}^{2}$ to $1.7 \mathrm{~A} / \mathrm{cm}^{2}$ is seen in the case of $\mathrm{Cr}^{2+}$ ions where the flux increases nearly twenty times. Since the flux of $\mathrm{Cr}^{+}$ions increases only by $36 \%$, in the limit of high $j_{T}$ the flux of doubly charged metal ions surpasses that of singly charged metal ions. The amount of $\mathrm{Ar}^{+}$in the ion flux to the substrate is almost unaffected by changes in $j_{T}$. The doubly charged gas ions $\left(\mathrm{Ar}^{2+}\right)$ on the other hand, are characterized by the lowest intensity of all ions detected and as such are of a minor importance.

Interesting to note in the context of metal ion current increasing with increasing the peak target current density is the behavior of the film growth rate that is plotted in Fig. $2 \mathrm{~b}$ also as a function of $j_{T}$. When going from $0.1 \mathrm{~A} / \mathrm{cm}^{2}$ up to $1.7 \mathrm{~A} / \mathrm{cm}^{2}$ the flux of film-forming species (neutrals, singly- and doubly-charged $\mathrm{Cr}$ ions) decreases approximately three times. Numerous explanations were put forward to account for such loss of rate [17] with back attraction of metal ions being a dominant one in the case of $\mathrm{Cr}$ deposition in a CC800/9 coating plant [18]. 
Nonetheless, a significant increase in the metal ion flux to the substrate with simultaneous decrease in the material flux imply that the $\mathrm{Cr}$ ion-to-neutral ratio must increase several times with increasing peak target current density within the limits studied here.

\section{$B)$ Influence of the ion-to-neutral ratio on samples facing the target}

Fig. 3 shows the $\{110\}$ and $\{200\}$ pole figures for the most representative cases of samples facing the target during $\mathrm{Cr}$ deposition. The reference sample prepared by DCMS under similar conditions (not shown) is characterized by very strong <200> texture. In case of films grown by HIPIMS (cf. Fig. 3a-b), the out-of-plane orientation common for all the samples is $<110\rangle$ which is the thermodynamically favorable configuration of the case of bcc structure (the lowest energy close-packed planes). Apparently the highly metallic character of the HIPIMS Cr plasma with relatively high content of $\mathrm{Cr}^{2+}$ ions (with respect to DCMS - cf. Fig. 2) ensures effective energy transfer to the growing film to follow growth mode typical for Zone II, despite the fact that no intentional heating was used. This takes place even at the lowest value of the peak target current density, $j_{T}=0.1 \mathrm{~A} / \mathrm{cm}^{2}$. The extended structure zone diagram proposed by Anders et al. [19] can be used to interpret this result. Within this treatment, among other modifications of the original Thornton diagram, the linear pressure axis is replaced by a logarithmic axis for normalized energy that takes into account effects caused by the kinetic energy of bombarding particles. The energy supplied by incident particles (in the present case primarily $\mathrm{Cr}$ ions) contributes to a non-local heating and shifts the working point to higher homologous temperature. In this way Zone II growth is possible even with no intentional heating. 


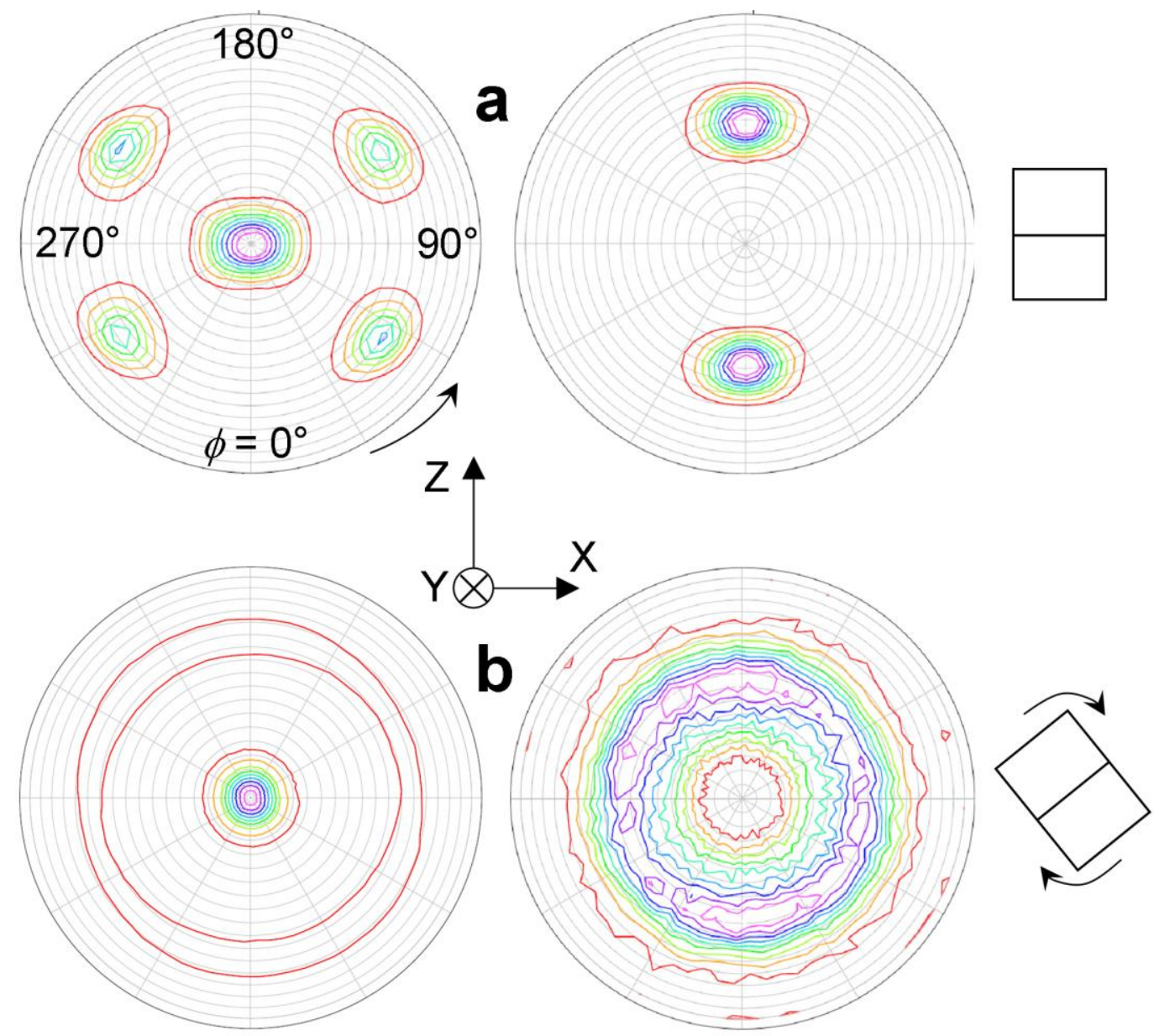

Fig. $3\{110\}$ (left) and $\{200\}$ (right) pole figure plots for a) $j_{T}=0.1 \mathrm{~A} / \mathrm{cm}^{2}$ and self-bias and b) $j_{T}$ $=1.7 \mathrm{~A} / \mathrm{cm}^{2}$ and $V_{S}=150 \mathrm{~V}$. Projections of a cube unit cells in the films are shown to the right. The target race track long direction is along $\mathrm{Z}$ axis (c.f. Fig. 1).

For the film grown at self-bias and under lower ion-to-neutral ratio, the $<110>$ outof-plane oriented grains exhibit a significant degree of biaxial alignment what is best seen in the corresponding $\{200\}$ pole figures (cf. Fig. 3a). The two signal maxima aligned along the $\phi=0^{\circ}$ $180^{\circ}$ axis indicate preferred in-plane orientation with the upper edge of the cube lined up with the $\mathrm{X}$ axis, i.e., perpendicular to the race track. The degree of the in-plane alignment, quantified by the full-width-at-half-maximum (FWHM) of the $\{200\}$ poles, amounts to $\pm 13^{\circ}$. This strong preference can be understood if one takes into account the spatial variations in the intensity of the 
material flux incident upon the substrate. That is caused by the large target dimensions $(50 \mathrm{~cm}$ long) and relatively short target-to-substrate distance $(\sim 5.7 \mathrm{~cm})$ resulting in highly asymmetric off-normal flux of the sputter-ejected species. As the target extends far out along the $\mathrm{Z}$ axis (as compared to the $\mathrm{X}$ axis) the $<110>$-oriented crystal grains with the sides of a $\mathrm{Cr}$ unit cell (cube) oriented as shown in the insets of Fig. 3 have the largest capture cross-section for the incident material flux and will thus overgrow grains with other in-plane orientations.

The situation is entirely different in the case of samples deposited at $j_{T}=1.7 \mathrm{~A} / \mathrm{cm}^{2}$ and $V_{S}=150 \mathrm{~V}$ (Fig. 3b). Although the $<110>$ out-of-plane orientation is retained, crystallites are randomly distributed in-plane of the substrate (typical fiber texture) as evident from the corresponding $\{200\}$ pole figure plot. Under these conditions (high ion-to-neutral ratio and high substrate bias) a majority of the material flux arrives at an angle that is close to the surface normal, despite the same geometry of the experimental set-up. This eliminates the factor responsible for the biaxial alignment discussed above for the $j_{T}=0.1 \mathrm{~A} / \mathrm{cm}^{2}$ case, as the capture cross-section for the incoming material flux becomes independent of the in-plane orientation of the $<110>$ out-of-plane oriented grains.

Since the dominating ionization mechanism in the case of HIPIMS plasma is electron impact ionization [20] the original momentum of the sputter-ejected neutrals is conserved in the ionization event. Therefore, in the hypothetical case of no electric fields being present, both neutrals and the corresponding ions (i.e., ions that got sputter-ejected as neutrals from the same portion of the target and become ionized on the way to the substrate) hit the surface at the same incidence angle, $\alpha$, from the surface normal. However, in real life situation, 
even if no intentional bias is used on the substrate, all ions will get deflected by inherently present potentials and arrive at the angle, $\beta$, such that $\beta<\alpha$. The effect will be further magnified by the application of substrate bias. The change in the incidence angle of ions with respect to neutrals emitted from the same portion of the race track can be estimated using the fact that only the momentum component along the surface normal (electric field direction) gets modified. Assuming the colissionless sheath ${ }^{21}$ simple geometrical considerations lead to the relationship between angles $\alpha$ and $\beta$ in the form

$$
\tan (\beta)=\frac{\sin (\alpha)}{\cos (\alpha)+\sqrt{\frac{q\left(V_{s}+V_{P}\right)}{E_{i}}}},
$$

where $E_{i}$ denotes the original energy of an ion, $q$ is the ion charge state and $V_{P}$ stands for plasma potential that in the case of HIPIMS is a time-dependent quantity. As the ion energy increases the term under square root in the denominator of Eq. (2) decreases and eventually in the limit of very high ion energy $\left(E_{i} \rightarrow \infty\right)$ the relation becomes $\alpha \approx \beta$, which is intuitively correct. In the case of deposition performed at low $j_{T}$ and self-bias conditions, the material flux is dominated by neutrals that travel along the line of sight between the emitting portion of the target and the substrate. Therefore, the incidence angle $\alpha$ is entirely determined by the geometrical set up and varies here between $22^{\circ}$ and $76^{\circ}$. The lower limit corresponds to neutrals sputter-ejected from the portions of the target closest to the substrate that move in the X-Y plane (cf. Fig. 1), whereas the upper limit applies to material emitted from the most distant portion of the target and moving in the $\mathrm{Y}-\mathrm{Z}$ plane (in the direction perpendicular to the plane of Fig. 1). In the case of a highly ionized plasma $\left(j_{T}=1.7 \mathrm{~A} / \mathrm{cm}^{2}\right)$ with the average $\mathrm{Cr}^{+}$ion energy during the pulse reaching $28 \mathrm{eV}$ [14], the ion incidence angle $\beta_{C r^{+}}$calculated using Eq. (2) for $V_{S}=150 \mathrm{~V}$ and $V_{P}=0 \mathrm{~V}$, varies from $7^{\circ}$ to $21^{\circ}$. 
For doubly charged $\mathrm{Cr}$ ions that dominate in the limit of high $j_{T}$ (the average $\mathrm{Cr}^{2+}$ ion energy of $22 \mathrm{eV}$ [14]), the incidence angle $\beta_{C r^{2+}}$ is between $5^{\circ}$ and $14^{\circ}$. Note that in real-life situation deflection should be even larger due to $V_{P}>0 \mathrm{~V}$. It is well-known that plasma potential during HIPIMS discharge can temporary reach tenths of Volts. This will of course further affect trajectory of all ions bringing them even closer to the substrate normal. Since in the limit of high $j_{T}$ neutrals constitute a minor portion in the material flux to the substrate (cf. discussion in the previous section and Fig. 2) it becomes evident that the off-normal flux is absent as nearly all deposits arrive at an angle smaller than $20^{\circ}$. In consequence, the $\langle 110\rangle$ out-of-plane oriented grains possess random in-plane distribution.

The effect of bias voltage on the in-plane alignment is further illustrated in Fig. 4 where the intensity of the diffracted signal is plotted along the azimuthal coordinate $\phi$ for different values of $V_{S}$. Two extreme cases of the lowest (Fig. 4a) and the highest (Fig. 4b) peak target current density are shown. As can be seen the best in-plane alignment characterized by the narrow peak at $\phi=180^{\circ}$ is obtained at the self-bias voltage. Even in the case of $j_{T}=1.7 \mathrm{~A} / \mathrm{cm}^{2}$ (cf. black plot in Fig. 4b) the film possess clear in-plane orientation (FWHM of $\pm 24^{\circ}$ ). The latter is a consequence of the fact that low electric field in the case of self-biased substrate $\left(V_{S}<10 \mathrm{~V}\right.$ during the pulse) has only a minor effect on the trajectory of incoming ions (cf. Eq. (2)). Therefore, the ion content in the flux plays a minor role for film texture formation. The difference between deposition performed under low and high $j_{T}$ becomes more evident at higher bias voltage, i.e., when the ionized portion of the flux can be steered effectively to arrive close to the surface normal. In general, as $V_{S}$ increases the in-plane alignment degrades. However, the effect is by far more pronounced in the case of $j_{T}=1.7 \mathrm{~A} / \mathrm{cm}^{2}$ where for $V_{S}>100 \mathrm{~V}$ random distribution 
results. In contrast, films prepared at $j_{T}=0.1 \mathrm{~A} / \mathrm{cm}^{2}$ thus at the lowest ion-to-neutral ratio, exhibit some degree of preferential in-plane orientation even at $V_{S}=150 \mathrm{~V}$. Clearly, in the later case the ionized fraction is too low to dominate the off-normal flux of neutrals.

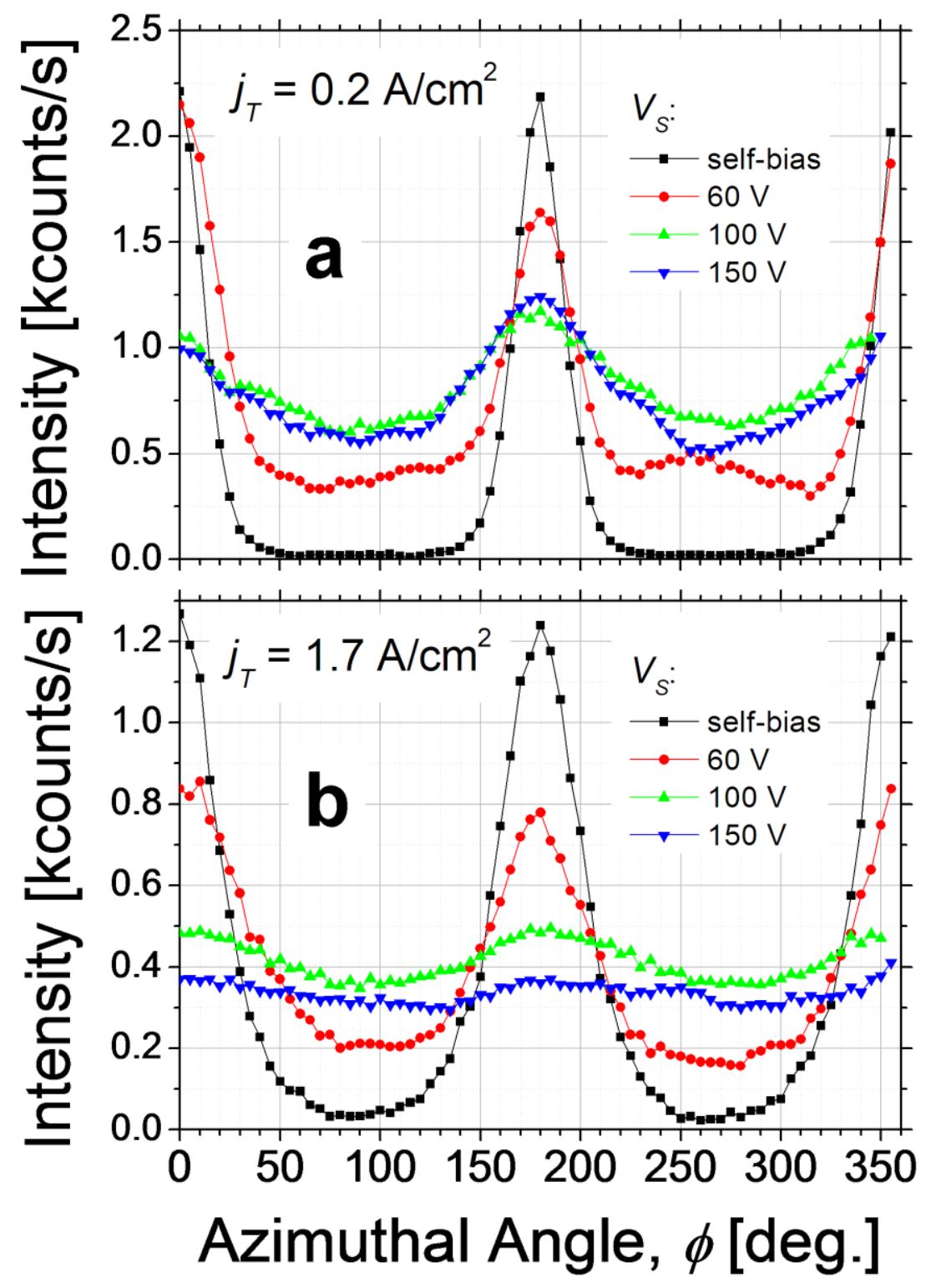

Fig. 4 Influence of bias voltage on the intensity of the diffracted signal along the azimuthal angle $\phi:$ A) $j_{T}=0.1 \mathrm{~A} / \mathrm{cm}^{2}$ and B) $j_{T}=1.7 \mathrm{~A} / \mathrm{cm}^{2}$.

For completeness, we emphasize that no biaxial alignment was observed for DCMS reference samples prepared under the same process pressure and the average power of $3 \mathrm{~kW}$. This 
result is explained by the fact that DCMS films possess the <200> texture that makes them insensitive to any non-homogeneities in the sputtered flux, in contrast to the $\langle 110\rangle$-oriented HIPIMS films. Thus, the role of HIPIMS in obtaining the bi-axially oriented films is primarily an increased energy transfer to the growing film that leads to the $\langle 110\rangle$ texture which, in turn, makes the crystallites sensitive to the geometry of the system.

\section{C) Influence of the ion-to-neutral ratio on inclined samples}

Figure 5 shows a set of fracture cross-section SEM micrographs from films deposited on substrates tilted by $90^{\circ}$ degrees from the target normal direction (samples denoted as "B" in Fig. 1). Fig. 5a shows the reference DCMS film whereas the rest of micrographs is for samples prepared by HIPIMS at different peak target current density ranging from $0.1 \mathrm{~A} / \mathrm{cm}^{2}$ (Fig. 5b) to $1.7 \mathrm{~A} / \mathrm{cm}^{2}$ (Fig. 5f). In all cases the bias voltage was $150 \mathrm{~V}$. The inset in Fig. 5a defines the terminology used below. In order to determine the column tilt relative to the substrate plane $(\mathrm{X}-\mathrm{Y})$, fracturing was performed parallel and perpendicular to the incoming flux. No column tilt was found in the direction perpendicular to the flux (X-Z plane). Thus, the SEM micrographs shown in Fig. 5 (cross-section in the Y-Z plane) fully reflect the column tilt with respect to the substrate plane.

In the case of the reference sample prepared by DCMS (Fig. 5a) columns are highly tilted in the direction of incoming flux. It can also be noted that the resulting film is very porous from inter-columnar voids, resulting from self-shadowing phenomena. In the present experimental set up the flux incidence angle $\delta$ (cf. Eq. (1)) is not well-defined since the width of 
the race track is significant with respect to the target-substrate distance. Taking into account the curvature of the race track, and the cosine distribution of the sputtered material flux it can be estimated that $\delta$ varies effectively between $70^{\circ}$ and $78^{\circ}$, thus somewhat outside of the applicability limits of the tangent rule [7]. Nevertheless, in the case of reference DCMS sample the average column tilt $\gamma$ is $57^{\circ}$ which upon application of the tangent rule (Eq. (1)) yields $\delta=$ $72^{\circ}$, i.e., in reasonable agreement with the estimates above.
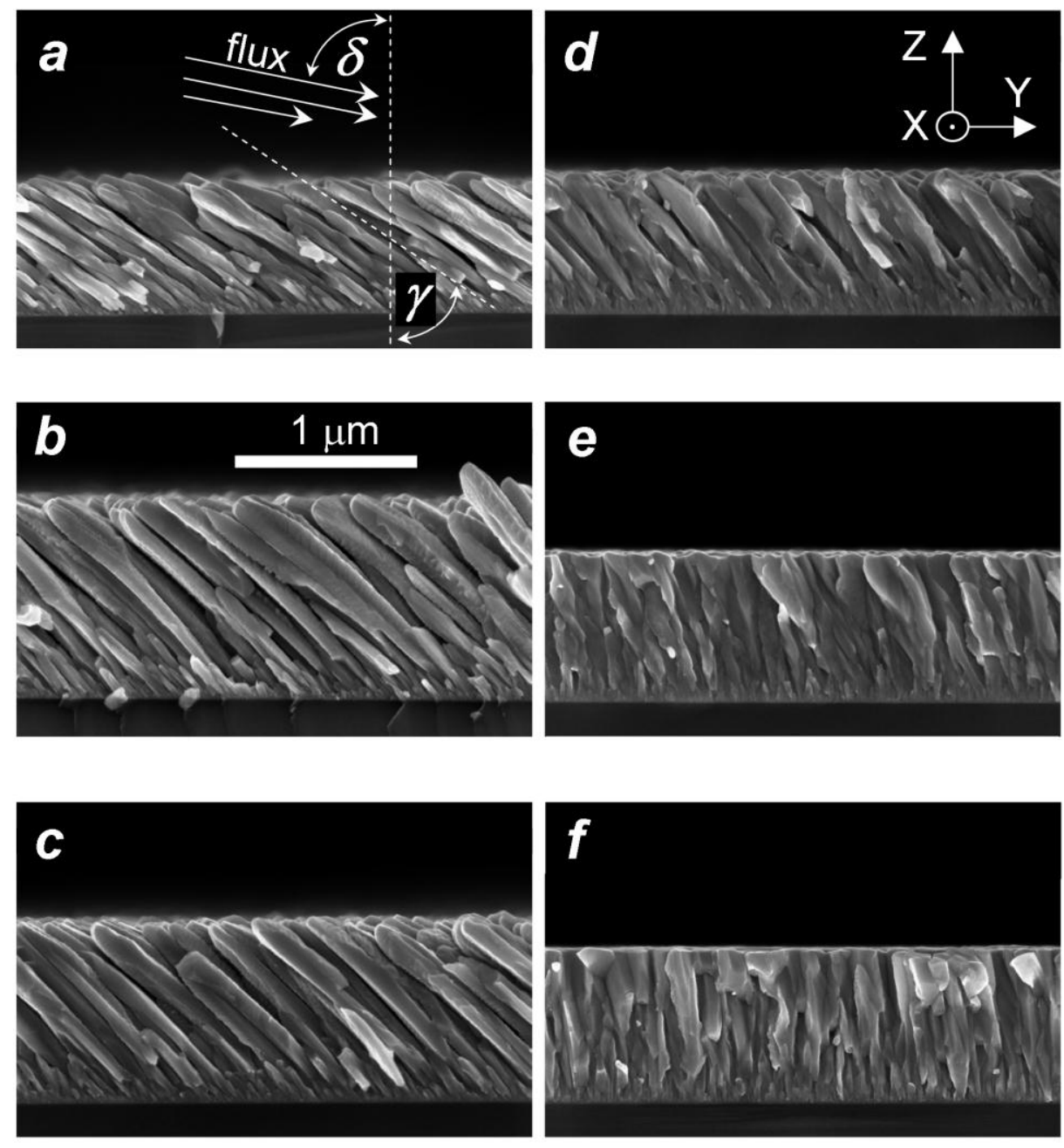

Fig. 5 Cross sectional SEM micrographs of films deposited on substrates oriented orthogonally to the target: a) DCMS reference, thereafter HIPIMS samples for increasing peak target current density b) $j_{T}=0.1 \mathrm{~A} / \mathrm{cm}^{2}$, c) $j_{T}=0.2 \mathrm{~A} / \mathrm{cm}^{2}$, d) $j_{T}=0.6 \mathrm{~A} / \mathrm{cm}^{2}$, e) $j_{T}=0.9 \mathrm{~A} / \mathrm{cm}^{2}, \mathrm{f}$ ) $j_{T}=1.7 \mathrm{~A} / \mathrm{cm}^{2}$. The inset in the upper-left graph self-explains definition of angles $\delta$ and $\gamma$ discussed in the text. The scale is the same for all micrographs. 
The series of SEM micrographs shown in Fig. 5b-f illustrates microstructure evolution for HIPIMS films upon increasing peak target current density, $j_{T}$. Already at the lowest value of $j_{T}=0.1 \mathrm{~A} / \mathrm{cm}^{2}$ (cf. Fig. 5b) changes with respect to the DCMS sample are noticeable the column tilt is reduced from $57^{\circ}$ to $50^{\circ}$ and the average column width increases. However, the film is still very porous and possesses high surface roughness. As the density of peak target current increases further, the column tilt gradually decreases, the surface flattens out, and voids are closing which make the film denser. Eventually, at the highest $j_{T}$ of $1.7 \mathrm{~A} / \mathrm{cm}^{2}$ (cf. Fig. 5f) columns grow close to the surface normal and film microstructure is very similar to that obtained on the substrate facing the target. This point is further verified by the plan view SEM images shown in Fig. 6 (substrate tilt angle $0^{\circ}$ vs. $90^{\circ}$ ) that reveal very similar surface topography for both types of samples. Fig. 7 summarizes the column tilt evolution as a function of the peak target current density. It can be noted that it is only the DCMS sample that follows the tangent rule. Otherwise, deviation from Eq. (1) increases with increasing $j_{T}$.
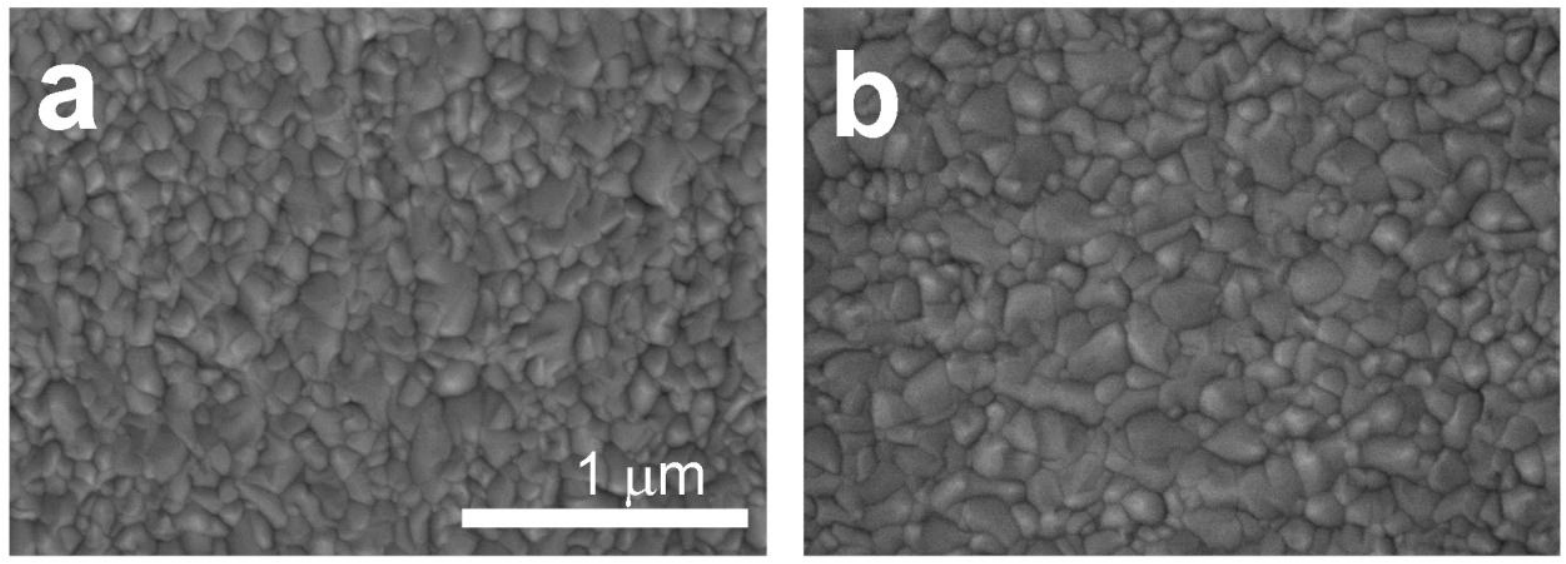

Fig. 6 Plan-view SEM images from films deposited at $j_{T}=1.7 \mathrm{~A} / \mathrm{cm}^{2}$; a) film on substrate facing the target and b) film on substrate tilted by $90^{\circ}$ with respect to the target.

The trends described above are the consequence of changes in the ion-to-neutral ratio with increasing $j_{T}$. The column tilt decreases with increasing ion-to-neutral ratio, as the 
direction of the incoming material flux is no longer determined exclusively by the substrate inclination angle. Again, Eq. (2) can be used to calculate the incidence angle for the ionized portion of the material flux impinging on the tilted substrate and, knowing that, the expected column tilt angle can be estimated from the tangent rule. The curves in Fig. 7 denoted as $\gamma_{\mathrm{Cr}^{+}}$and $\gamma_{C r^{2+}}$ represent the expected column tilt angle for $\mathrm{Cr}^{+}$and $\mathrm{Cr}^{2+}$ ions in case of purely ionic deposition. It can be observed that as $j_{T}$ increases the column tilt angle moves away from the "tangent rule" zone towards the predictions for the ionic deposition. Eventually at $j_{T}=1.7 \mathrm{~A} / \mathrm{cm}^{2}$ the angle $\gamma$ measured from the corresponding SEM micrograph (Fig. 5f) falls within the limits of ionic deposition indicating that flux to the substrate is highly dominated by ions. This is in agreement with results obtained for films facing the target where the effect of highly-ionized flux was manifested by randomization of the in-plane distribution. One may expect that the ion-toneutral ratio can be even higher on inclined substrates due to lower capture cross-section for neutrals that get deposited along the line of sight to the race track. This may account for the fact that at $j_{T}=1.7 \mathrm{~A} / \mathrm{cm}^{2} \gamma$ assumes values predicted for $100 \%$ ionization level.

Kuratani et al. [22] reported a decrease (or even a complete elimination) of the column tilt during growth of $\mathrm{Cr}$ film with simultaneous irradiation with high-energy normalincident Ar ions. This effect was achieved for $\mathrm{Ar}^{+}$ion energy of $20 \mathrm{keV}$ and $\mathrm{Ar}^{+}$-to-Cr adatom ratio as low as 0.033 and explained by enhanced adatom mobility resulting from collisions with incident Ar ions. Despite the similar final effect of the reduced column tilt the underlying physical mechanisms should be different in the present case considering that our $\mathrm{Ar}^{+}$ions possess significantly lower energy $(\sim 160 \mathrm{eV})$ and therefore are not able to induce effects described in Ref. [22]. In contrast to the work of Kuratani et al. the ion flux during HIPIMS is dominated by 
film-forming ions what allows for a direct control over the incidence angle, and elimination of the column tilt without the need of an additional ion source.

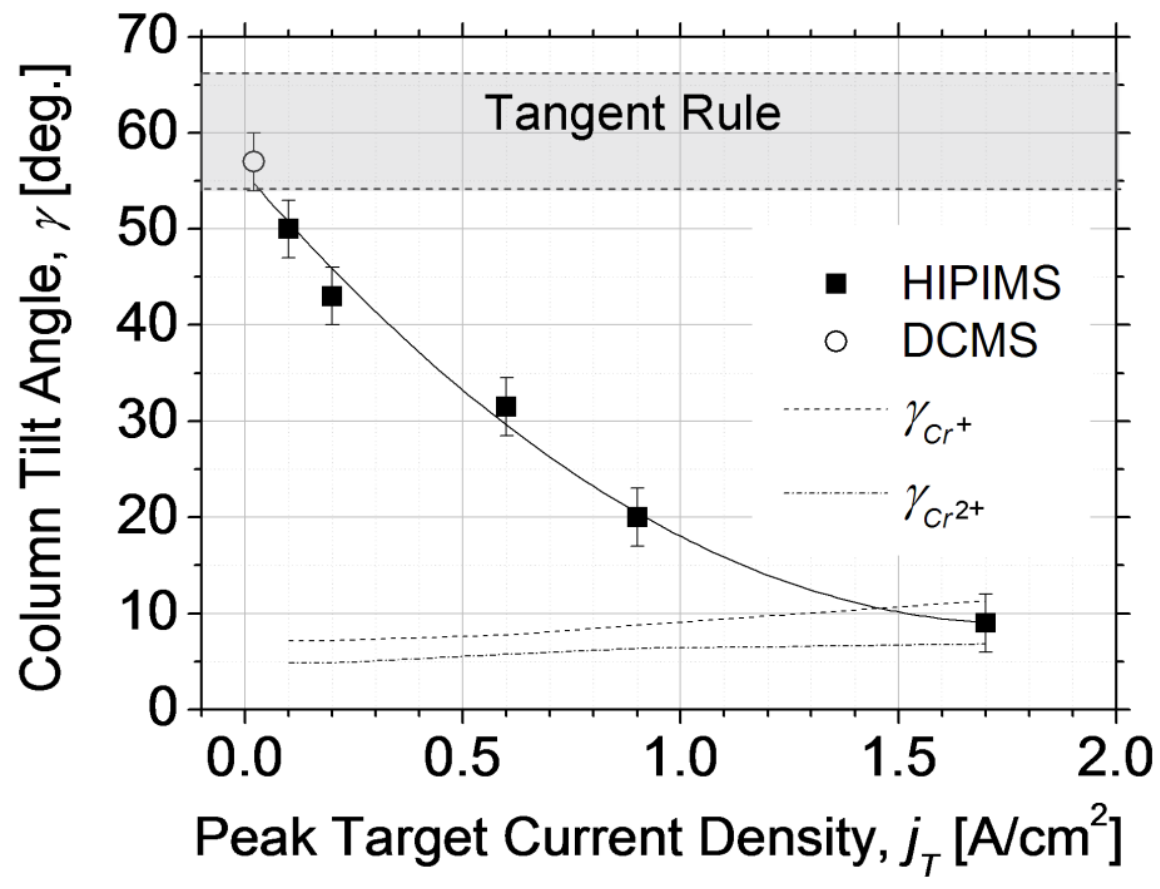

Fig. 7 Column tilt angle $\gamma$ as a function of the peak target current density, $j_{T}$. The data point for DCMS sample corresponds to the average current density. The grayed area represents the validity region of the tangent rule for $\delta$ varying between $70^{\circ}$ and $78^{\circ}$. Two curves, $\gamma_{C^{+}}$ and $\gamma_{\mathrm{Cr}^{2+}}$ represent expected column tilt angle in case of purely ionic deposition with $\mathrm{Cr}^{+}$ and $\mathrm{Cr}^{2+}$ ions, respectively. The uncertainty in the angle $\delta$ has a minor effect on these plots (less than $1^{\circ}$ for $70^{\circ}<\delta<78^{\circ}$ ). The raising trend with increasing $j_{T}$ has to do with the fact that the average ion energy increases at higher peak target current density.

The $\{110\}$ and $\{200\}$ pole figures for obliquely-deposited samples are shown in Fig. 8 for different values of $j_{T}$ increasing from $0.1 \mathrm{~A} / \mathrm{cm}^{2}$ (Fig. 8a) up to $1.7 \mathrm{~A} / \mathrm{cm}^{2}$ (Fig. 8d). Common for all samples is a certain degree of in-plane alignment. At lower peak target current density, $j_{T}=0.1-0.2 \mathrm{~A} / \mathrm{cm}^{2}$ deposition on substrates inclined by $90^{\circ}$ with respect to the target results in the $<111>$ out-of-plane orientation (cf. Fig. 8a) with the main crystal axis tilted by $8^{\circ}$ in the direction of incoming flux. Note that there is no direct correlation between the column tilt angle $\left(\gamma=50^{\circ}\right)$ and the preferred grain orientation, rather than that both are consequences of the 
oblique deposition. The <111> out-of-plane oriented grains are faceted by the fastest growing $\{100\}$ planes resulting in triangular plan view shape (cf. projected view in Fig. 8a). For such case, the model presented in Ref. [1] predicts that most favorable in-plane orientation is realized when the angle between the direction of incoming flux and triangle side is either $30^{\circ}$ or $90^{\circ}$. As can be seen from Fig. 8a that can in fact be realized at lower values of $j_{T}$. The mean deviation from the in-plane alignment axis can be derived from the FWHM of the $<200>$ pole pointing towards the incident flux and amounts to $\pm 13^{\circ}$. Similar type of bi-axial alignment for $\langle 111\rangle$ outof-plane oriented grains was also observed for reactive sputter deposition of TiN on tilted substrates [23]. With increasing peak target current density (hence the ion-to-neutral ratio), films acquire the <110> out-of-plane texture, characteristic of deposition on substrates facing the target, already at $j_{T}=0.6 \mathrm{~A} / \mathrm{cm}^{2}$ (Fig. $8 \mathrm{~b}$ ). The $\langle 110\rangle$ crystal axis is tilted away from the direction of incoming flux by $6^{\circ}$, showing again no correlation to the column tilt angle of $32^{\circ}$. The FWHM of the $\langle 200\rangle$ pole pointing towards the incoming flux increased to $\pm 17^{\circ}$ indicating slightly worse in-plane alignment than at $j_{T}=0.1 \mathrm{~A} / \mathrm{cm}^{2}$. The corresponding SEM micrograph indicates that the film is still porous and rough (cf. Fig. 5d). Thus, the effect of increased ion-toneutral ratio is first seen by the reduced column tilt and changes in the film texture before any modifications in morphology take place. When going to even higher $j_{T}$ of $0.9 \mathrm{~A} / \mathrm{cm}^{2}$ (Fig. 8c) the $\langle 110\rangle$ out-of-plane orientation is preserved (with the $\langle 110\rangle$ axis tilted by $16^{\circ}$ away from the flux), however, the direction of the preferred in-plane alignment rotates by $90^{\circ}$ with respect to the $j_{T}=0.6 \mathrm{~A} / \mathrm{cm}^{2}$ case. In this way the film texture resembles that of the samples facing the target during deposition at lower substrate bias (cf. Fig. 3a). As the ion content in the deposition flux increases, the tendency for the in-plane alignment gets reduced what is clearly indicated by the broadening of the $\langle 200\rangle$ poles $\left(\mathrm{FWHM}\right.$ of $\left.\pm 34^{\circ}\right)$. As could be expected at the highest value of 
peak target current density, $j_{T}=1.7 \mathrm{~A} / \mathrm{cm}^{2}$ (cf. Fig. 8d), the in-plane alignment is even less pronounced $\left(\mathrm{FWHM}= \pm 42^{\circ}\right)$ and the film texture gets close to that of the corresponding film deposited on substrate facing the target (cf. Fig. 3b).

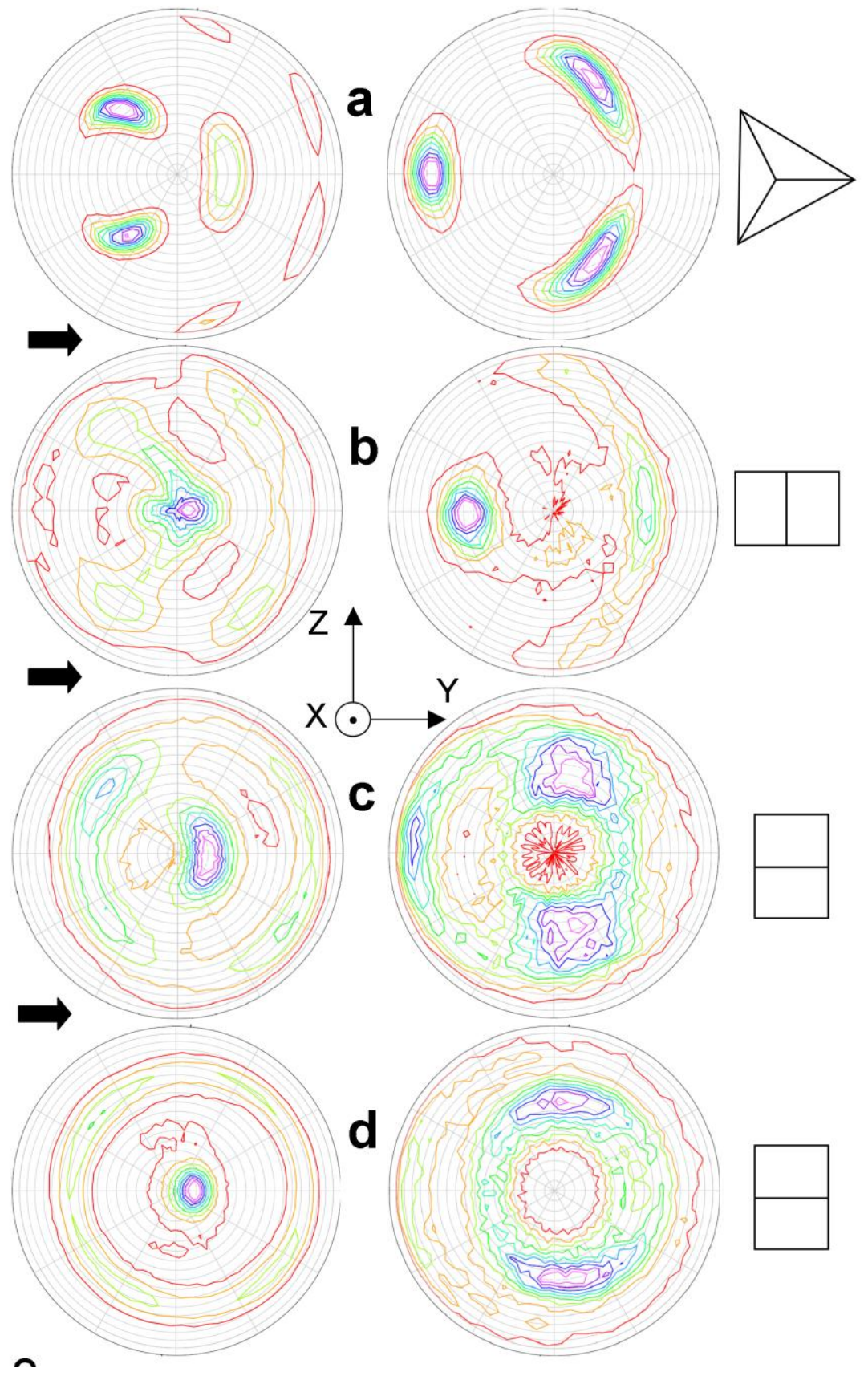

Fig. $8\{110\}$ (left) and $\{200\}$ (right) pole figure plots for films deposited on tilted substrates. The following cases are shown: a) $j_{T}=0.1 \mathrm{~A} / \mathrm{cm}^{2}$, b) $j_{T}=0.6 \mathrm{~A} / \mathrm{cm}^{2}$, c) $j_{T}=0.9 \mathrm{~A} / \mathrm{cm}^{2}$, d) $j_{T}=1.7$ $\mathrm{A} / \mathrm{cm}^{2}$. The arrows mark the direction of the incoming flux. Projections of a cube unit cells in the films are shown to the right. 
Finally, Fig. 9 shows photographs taken of two series of samples; a) deposited on substrate facing the target and b) deposited on substrates orthogonally-mounted with respect to the target with different peak target current density. It can be seen that obliquely-deposited films change color as the columns tilt away from surface normal leading to higher surface roughness and increased light scattering. This simple visual inspection may be used for quick qualitative verification of the ionization degree in the sputtered material flux arriving at the substrate.

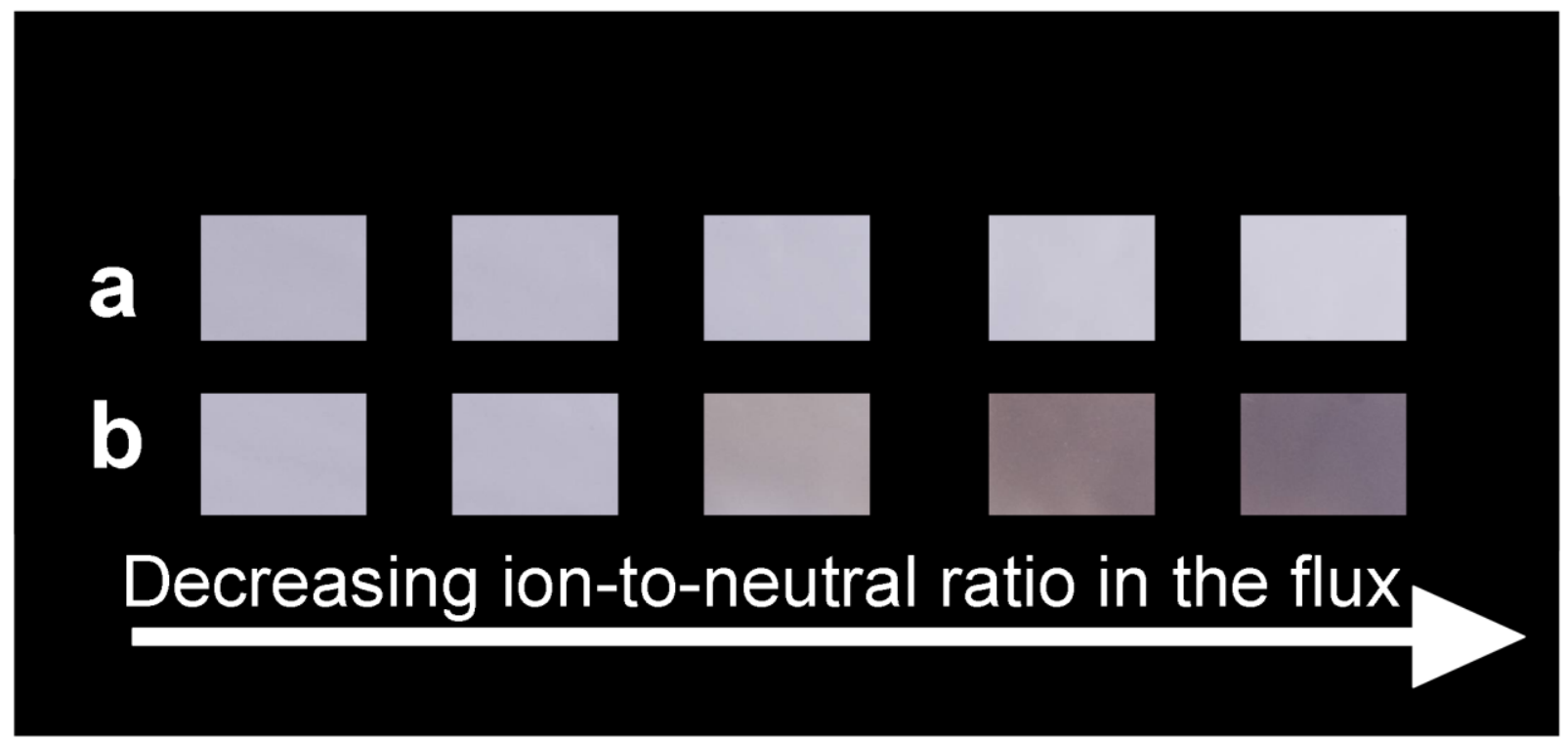

Fig. 9 Photograph showing two sets of samples: a) samples facing the target during deposition and b) corresponding samples grown on substrates tilted by $90^{\circ}$ with respect to the target. The peak target current density (hence the ion-to-neutral ratio) decreases from left to right.

\section{CONCLUSIONS}

HIPIMS was used to grow chromium thin films on substrates facing and orthogonal to the target. It is shown that with increasing peak target current density, $j_{T}$, from $0.1 \mathrm{~A} / \mathrm{cm}^{2}$ to 1.7 $\mathrm{A} / \mathrm{cm}^{2}$ the $\mathrm{Cr}$ ion-to-neutral ratio in the flux to the substrate greatly increases as a consequence of 
nearly three-fold increase in the metal ion flux $\left(\mathrm{Cr}^{+}\right.$and $\left.\mathrm{Cr}^{2+}\right)$ accompanied by a subsequent decrease in the film growth rate by approximately the same factor.

Using the fact that the ion-to-neutral ratio may be steered on purpose by adjusting $j_{T}$, the influence of the relative ion content in the material flux impinging on the substrate on those aspects of the film growth that are directly related to the geometry of the target-substrate configuration was studied. At low ion-to-neutral ratio films grown on substrates facing the target possess <110> out-of-plane orientation and exhibit high degree of in-plane alignment. The latter is assigned to the presence of the asymmetrical off-normal flux of sputtered species in the direction parallel to the longer side of the rectangular target that favors growth of grains with maximum capture cross section for incoming species. The biaxial alignment somewhat degrades at high bias voltage as the ionic portion of the incoming flux gets affected by the electric field and arrives close to the surface normal. However, the completely random distribution (fiber texture) can only be obtained if the deposition is performed at high peak target current density (and substrate bias larger than $100 \mathrm{~V}$ ), i.e., under conditions when the incoming flux is dominated by ions.

In the case of films grown on substrates orthogonal to the target the line-of-sight deposition typically leads to film columns tilting in the direction of the incoming flux. Again, similar to the case of the deposition on substrates facing the target, the sufficiently high ionization of sputtered flux leads to the situation where geometrical restrictions, dominant in conventional DCMS, play a minor (or no) roll in determination of film texture and microstructure. The large column tilt characteristic for growth at low $j_{T}$, decreases with increasing ion content in the flux and almost disappears at the highest value of $j_{T}$. The later indicates that 
material flux to the substrate is highly ionized so that deposition takes place along substrate normal despite the high inclination angle. Thus, in the limit of high peak target current density the artifacts of the conventional PVD, resulting from the line-of-sight deposition, are eliminated and the film growth proceeds to large extent independently of substrate orientation.

These results are of particular importance for coatings applied to multi-shaped

objects or surfaces that are not facing the target during deposition, e.g., the rake face of the cutting tool that stays perpendicular to the target for the whole deposition sequence.

\section{ACKNOWLEDGEMENTS}

The financial support from the European Research Council (ERC) through an Advanced Grant is acknowledged. We thank the staff at CemeCon AG and at the Tandem Laboratory, Uppsala University, for technical support. 


\section{REFERENCES}

[1] S. Mahieu, P. Ghekiere, D. Depla, R. De Gryse Biaxial alignment in sputter deposited thin films Thin Solid Films 515 (2006) 1229-1249

[2] R. M. Bradley, J. M. E. Harper, and D. A. Smith Theory of thin-film orientation by ion bombardment during deposition J. Appl. Phys. 60 (1986) 4160-4164

[3] P. Ghekiere, S. Mahieu, R. De Gryse, D. Depla, Structure evolution of the biaxial alignment in sputter-deposited $\mathrm{MgO}$ and $\mathrm{Cr}$, Thin Solid Films 515 (2006) 485-488

[4] J.F. Whitacre, S.M. Yalisove and J.C. Bilello, In-plane Texturing in sputtered films Texture Microstruct. 34 (2000) 91-103

[5] L. Abelmann, C. Lodder Oblique evaporation and surface diffusion Thin Solid Films 305 (1997) 1-21

[6] See, e.g., M. Ohring Materials Science of Thin Films, Academic Press; 2nd Ed. (October 29, 2001), p. 506

[7] Paritosh, D.J. Srolovitz Shadowing effects on the microstructure of obliquely deposited films, J. Appl. Phys. 91 (2002) 1963-72

[8] J. Alami, P. O. Å. Persson, D. Music, J. T. Gudmundsson, J. Bohlmark and U. Helmersson Ion-assisted physical vapor deposition for enhanced film properties on non-flat surfaces J. Vac. Sci. Technol. A 23 (2005) 1-3

[9] U. Helmersson, M. Lattemann, J. Bohlmark, A. P. Ehiasarian, J. T. Gudmundsson Ionized physical vapor deposition (IPVD): A review of technology and applications Thin Solid Films 513 (2006) 1-24

[10] K. Sarakinos, J. Alami, S. Konstantinidis High power pulsed magnetron sputtering: A review on scientific and engineering state of the art Surf. Coat. Tech. 204 (2010) 1661-1684

[11] J. Bohlmark, M. Östbye, M. Lattemann, H. Ljungcrantz, T. Rosell, U. Helmersson Guiding the deposition flux in an ionized magnetron discharge Thin Solid Films 515 (2006) 1928-31

[12] http://www.cemecon.de/coating technology/2 coating units/25 cc 800sup_sup_9 hipims/index eng.html, accessed in October 2010

[13] The reference measurements performed in the DCMS mode indicated that as long as the ion-to-neutral ratio and the substrate bias is kept constant, similar film texture (and column tilt) is achieved, quite independently of the flux incident on the substrate (within the experimental limits tested).

[14] G. Greczynski and L. Hultman Time and energy resolved ion mass spectroscopy studies of the ion flux during high power pulsed magnetron sputtering of $\mathrm{Cr}$ in $\mathrm{Ar}$ and $\mathrm{Ar} / \mathrm{N}_{2}$ atmospheres Vacuum 84 (2010) 1159-1170

[15] M. Mayer Nucl. Instrum. Meth. B 194 (2002) 177 (http://www.simnra.com/).

[16] D. Lundin, N. Brenning, D. Jadernas, P. Larsson, E. Wallin, M. Lattemann, M. A. Raadu, and U. Helmersson Transition between the discharge regimes of high power impulse magnetron sputtering and conventional direct current magnetron sputtering Plasma Sources Science and Technology 18, 045008 (6 pp.) (2009).

[17] A. Anders Deposition rates of high power impulse magnetron sputtering: Physics and economics J. Vac. Sci. Technol. A 28 (2010) 783

[18] G. Greczynski, J. Jensen, J. Böhlmark and L. Hultman Microstructure control of $C r N_{x}$ films during high power impulse magnetron sputtering Surf. Coat. Tech. 205 (2010) 118-130

[19] A. Anders A structure zone diagram including plasma-based deposition and ion etching Thin Solid Films 518 (2010) 4087-4090

[20] J.T. Gudmundsson The High Power Impulse Magnetron Sputtering Discharge: The Ionization Mechanism Society of Vacuum Coaters 49th (2006) Annual technical Conference Proceedings, 1-5 (2006).

[21] Since very little is known about the basic parameters that determine the sheath widths in the case of HIPIMS discharges one can only use arguments from the classical processing discharges. At $3 \mathrm{mTorr}$ (process pressure used in our experiment) the mean free path for ion collisions with Ar molecules is $\sim 1.6 \mathrm{~cm}$, i.e., somewhat larger than the sheath width in the case of high substrate bias (Child law sheath is typically less than $1 \mathrm{~cm}$ ) and lot larger than the sheath width for floating substrate (less than $1 \mathrm{~mm}$ ). Within this simplified classical treatment sheaths can thus be treated as colissionless.

[22] N. Kuratani, A. Ebe, K. Ogata, I. Shimizu, Y. Setsuhara and S. Miyake Fundamental study of ion-irradiation effects on the columnar growth of chromium films prepared by ion-beam and vapor deposition J. Vac. Sci. Technol. A 19 (2001) 153-157 
[23] S. Mahieu, P. Ghekiere, G. De Winter, R. De Gryse, D. Depla, G. Van Tendeloo, O.I. Lebedev Biaxially aligned titanium nitride thin films deposited by reactive unbalanced magnetron sputtering Surf. Coat. Tech. 200 (2006)

2764- 2768 